PROCEEDINGS OF THE

AMERICAN MATHEMATICAL SOCIETY

Volume 128, Number 9 , Pages 2621-2626

S 0002-9939(00)05442-3

Article electronically published on February 29, 2000

\title{
ON SINGULAR CRITICAL POINTS OF POSITIVE OPERATORS IN KREIN SPACES
}

\author{
BRANKO ĆURGUS, AURELIAN GHEONDEA, AND HEINZ LANGER
}

(Communicated by David R. Larson)

\begin{abstract}
We give an example of a positive operator $B$ in a Krein space with the following properties: the nonzero spectrum of $B$ consists of isolated simple eigenvalues, the norms of the orthogonal spectral projections in the Krein space onto the eigenspaces of $B$ are uniformly bounded and the point $\infty$ is a singular critical point of $B$.
\end{abstract}

An operator $A$ in the Krein space $(\mathcal{K},[\cdot, \cdot])$ is said to be positive if $[A x, x]>0$ for all nonzero $x$ in the domain of $A$. A bounded positive operator $A$ in the Krein $\operatorname{space}(\mathcal{K},[\cdot, \cdot])$ has a projection valued spectral function $E$ with 0 being its only possible critical point (see [1, Theorem IV.1.5] or [5, Section II.3.]). Recall that, by [5, Proposition 5.6], the condition

$$
\|E((-\infty, \alpha])\| \leq C_{-}<\infty \text { for all } \alpha<0
$$

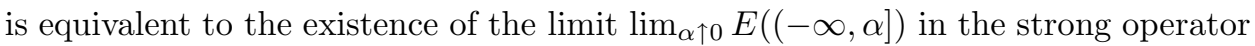
topology. Similarly,

$$
\|E([\beta, \infty))\| \leq C_{+}<\infty \text { for all } \beta>0
$$

is equivalent to the existence of the $\operatorname{limit}_{\beta \downarrow 0} E([\beta,+\infty))$ in the strong operator topology. Since 0 is not an eigenvalue of a positive operator $A$, [5, Proposition 3.2] implies that (11) and (2) are equivalent. Also, if 0 is a critical point, it is said to be regular if one of the conditions (11) or (2) is fulfilled. If the critical point 0 is not regular, it is called singular.

In the sequel the operator $A$ considered will have a discrete spectrum outside 0 . Examples of bounded positive operators in $\mathcal{K}$ having 0 as a singular critical point can be constructed as follows (see also the examples in [2, Section 1], [3], 4]). Consider a sequence of two-dimensional Krein spaces $\mathcal{K}_{n}=\mathbb{C}^{2}$ with fundamental symmetry $J_{n}=\left(\begin{array}{cc}1 & 0 \\ 0 & -1\end{array}\right)$ and positive operators $A_{n}$ in $\mathcal{K}_{n}$; denote by $\lambda_{n}^{+}\left(\lambda_{n}^{-}\right.$, respectively $)$ its positive (negative, respectively) eigenvalues and by $P_{n}^{+}\left(P_{n}^{-}\right.$, respectively) the orthogonal (in $\mathcal{K}_{n}$ ) projection onto the corresponding eigenspace.

Received by the editors October 15, 1998.

2000 Mathematics Subject Classification. Primary 47B50, 46C50.

Key words and phrases. Krein space, definitizable operator, critical point.

The third author was supported by Fonds zur Förderung der wissenschaftlichen Forschung of Austria, Project P 12176 MAT. 
If $A_{n}$ is chosen such that $\left\|A_{n}\right\| \leq C$ for all $n, \lambda_{n}^{+} \downarrow 0, \lambda_{n}^{-} \uparrow 0,\left\|P_{n}^{ \pm}\right\| \rightarrow \infty$ if $n \rightarrow \infty$, then $A=\bigoplus_{n=1}^{\infty} A_{n}$ is a bounded positive operator in $\mathcal{K}=\bigoplus_{n=1}^{\infty} \mathcal{K}_{n}$ having 0 as a singular critical point. Evidently,

$$
\sigma(A)=\left\{\lambda_{n}^{+}, \lambda_{n}^{-} \mid n \in \mathbb{N}\right\} \cup\{0\},
$$

and $\left\|E\left(\left\{\lambda_{n}^{ \pm}\right\}\right)\right\| \rightarrow \infty$ if $n \rightarrow \infty$, that is, the eigenvectors $f_{n}^{+}, f_{n}^{-}$of $A$ corresponding to $\lambda_{n}^{+}$and $\lambda_{n}^{-}$, respectively, become arbitrarily close if $n$ is large.

The question arises whether or not 0 can be a singular critical point of a positive operator $A$ in $\mathcal{K}$ with discrete spectrum $\left\{\lambda_{n}^{+}, \lambda_{n}^{-} \mid n \in \mathbb{N}\right\}$ in $\mathbb{C} \backslash\{0\}$ if the projections $E\left(\left\{\lambda_{n}^{ \pm}\right\}\right)$are uniformly bounded. It is the aim of this note to show that the answer is yes: We will construct a bounded positive operator $A$ in a Krein space $\mathcal{K}$, such that the projections $E\left(\left\{\lambda_{n}^{ \pm}\right\}\right)$corresponding to the single eigenvalues are uniformly bounded but, nevertheless,

$$
\left\|E\left(\left\{\lambda_{1}^{ \pm}, \ldots, \lambda_{n}^{ \pm}\right\}\right)\right\| \longrightarrow \infty, \quad n \longrightarrow \infty .
$$

Our construction is based on the following two lemmas.

Lemma 1. Let $\mathcal{H}_{n}$ be an n-dimensional vector space with a positive definite scalar product $(\cdot, \cdot)$. Then there exist a basis $f_{n 1}, \ldots, f_{n n}$ of $\mathcal{H}_{n}$ and a positive contraction $S_{n}$ in $\mathcal{H}_{n}$ such that

$$
0<1 \leq\left\|f_{n k}\right\| \leq 2, \quad\left\|S_{n}^{-1}\right\|=n, \quad\left(S_{n} f_{n j}, f_{n k}\right)=\delta_{j k}, j, k=1, \ldots, n .
$$

Proof. Let $e_{n 1}, \ldots, e_{n n}$ be an orthonormal basis of $\mathcal{H}_{n}$, let $T_{n}$ be the selfadjoint transformation in $\mathcal{H}_{n}$ given by $T_{n} e_{n 1}=\sqrt{n} e_{n 1}, T_{n} e_{n j}=e_{n j}, j=2, \ldots, n$, and put $S_{n}=T_{n}^{-2}$. Evidently, $S_{n}$ is a positive selfadjoint contraction in $\mathcal{H}_{n}$, and $\min \sigma\left(S_{n}\right)=1 / n$. Therefore $\left\|S_{n}^{-1}\right\|=n$. Let $\left(u_{k 1} \ldots u_{k n}\right), k=1, \ldots, n$, be an orthonormal basis of the $n$-dimensional space of row vectors with components in $\mathbb{C}$, such that $u_{1 j}=1 / \sqrt{n}, j=1, \ldots, n$. Then $U=\left(u_{k j}\right)_{k, j=1}^{n}$ is a unitary matrix with $u_{1 j}=1 / \sqrt{n}, j=1, \ldots, n$. Put

$$
\phi_{n j}=\sum_{k=1}^{n} u_{k j} e_{n k}, \quad j=1, \ldots, n .
$$

Then $\phi_{n j}, j=1, \ldots, n$, is an orthonormal basis of $\mathcal{H}_{n}$ and

$$
\left\|T_{n} \phi_{n j}\right\|^{2}=n \frac{1}{n}+\sum_{k=2}^{n}\left|u_{k j}\right|^{2}=1+1-\frac{1}{n}, j=1, \ldots, n .
$$

Hence $1 \leq\left\|T_{n} \phi_{n j}\right\| \leq 2$. Let $f_{n j}=T_{n} \phi_{n j}, j=1, \ldots, n$. Then $1 \leq\left\|f_{n j}\right\| \leq 2$ and $\left(S_{n} f_{n j}, f_{n k}\right)=\left(\phi_{n j}, \phi_{n k}\right)=\delta_{j k}, j, k=1, \ldots n$. The lemma is proved.

Lemma 2. Let $(\mathcal{H},(\cdot, \cdot))$ be a separable Hilbert space and let $P$ be a positive, bounded and boundedly invertible operator in $\mathcal{H}$. Let $\phi_{j}, j \in \mathbb{N}$, be a Riesz basis of $\mathcal{H}$ such that $\left(P \phi_{j}, \phi_{k}\right)=\delta_{j k}, j, k \in \mathbb{N}$, and let $\lambda_{j} \in \mathbb{C}, j \in \mathbb{N}$, be a bounded sequence. Define the operator $A$ in $\mathcal{H}$ by $A \phi_{j}=\lambda_{j} \phi_{j}, j \in \mathbb{N}$. Then, $A$ can be extended by continuity to a bounded linear operator in $\mathcal{H}$ such that $\|A\| \leq$ $\sqrt{\|P\|\left\|P^{-1}\right\|} \sup \left\{\left|\lambda_{j}\right|, j \in \mathbb{N}\right\}$.

Proof. For a bounded and boundedly invertible positive operator $P$ we have

$$
\left\|P^{-1}\right\|^{-1}(x, x) \leq(P x, x) \leq\|P\|(x, x), \quad x \in \mathcal{H} .
$$


Since the vectors $\phi_{j}, j \in \mathbb{N}$, are orthonormal with respect to the inner product $(P \cdot, \cdot)$, it follows that

$$
(P A x, A x) \leq\left(\sup \left\{\left|\lambda_{j}\right|, j \in \mathbb{N}\right\}\right)^{2}(P x, x), \quad x \in \mathcal{H} .
$$

Combining (3) and (4) we get

$$
\begin{aligned}
\|A x\|^{2} & =(A x, A x) \leq\left\|P^{-1}\right\|(P A x, A x) \leq\left\|P^{-1}\right\|\left(\sup \left\{\left|\lambda_{j}\right|, j \in \mathbb{N}\right\}\right)^{2}(P x, x) \\
& \leq\left\|P^{-1}\right\|\|P\|\left(\sup \left\{\left|\lambda_{j}\right|, j \in \mathbb{N}\right\}\right)^{2}\|x\|^{2}
\end{aligned}
$$

and the lemma follows.

Theorem. There exist a Krein space $(\mathcal{K},[\cdot, \cdot])$ and a bounded positive operator $A$ in $\mathcal{K}$ with the following properties:

(a) The nonzero spectrum of $A$ consists of isolated simple eigenvalues.

(b) The point 0 is a singular critical point of $A$.

(c) The norms of the orthogonal projections in the Krein space $(\mathcal{K},[\cdot, \cdot])$ onto the eigenspaces of $A$ are uniformly bounded.

Proof. With the notation as in Lemma 1, choose $\mathcal{H}_{n}^{+}=\mathcal{H}_{n}^{-}=\mathcal{H}_{n}$. Let $\mathcal{K}_{n}=$ $\mathcal{H}_{n}^{+} \oplus \mathcal{H}_{n}^{-}$be the direct sum of the Hilbert spaces $\left(\mathcal{H}_{n}^{ \pm},(\cdot, \cdot)\right)$. The positive definite inner product on $\mathcal{K}_{n}$ is also denoted by $(\cdot, \cdot)$. All norms in $\mathcal{K}_{n}$ correspond to this inner product. Endow $\mathcal{K}_{n}=\mathcal{H}_{n}^{+} \oplus \mathcal{H}_{n}^{-}$with the indefinite inner product $[\cdot, \cdot \cdot]$ given by the fundamental symmetry $J_{n}=\left(\begin{array}{cc}I_{n} & 0 \\ 0 & -I_{n}\end{array}\right)$. Consider the operator $K_{n}^{+}=\left(I_{n}-S_{n}\right)^{1 / 2}$ acting from $\mathcal{H}_{n}^{+}$into $\mathcal{H}_{n}^{-}$as an angular operator in $\mathcal{K}_{n}$. Here $S_{n}$ is the operator constructed in Lemma1. Let $\mathcal{L}_{n}^{+}$be the graph of $K_{n}^{+}$in $\mathcal{K}_{n}=\mathcal{H}_{n}^{+} \oplus \mathcal{H}_{n}^{-}$. Then $\mathcal{L}_{n}^{+}$is an $n$-dimensional maximal positive subspace in $\mathcal{K}_{n}$. It is spanned by the vectors $\boldsymbol{f}_{n k}^{+}=\left(\begin{array}{c}f_{n k} \\ K_{n}^{+} f_{n k}\end{array}\right), k=1, \ldots, n$, and

$$
\begin{gathered}
{\left[\boldsymbol{f}_{n k}^{+}, \boldsymbol{f}_{n j}^{+}\right]=\left(f_{n k}, f_{n j}\right)-\left(K_{n}^{+} f_{n k}, K_{n}^{+} f_{n j}\right)=\left(S_{n} f_{n k}, f_{n j}\right)=\delta_{k j},} \\
\left\|\boldsymbol{f}_{n k}^{+}\right\|^{2}=\left\|f_{n k}\right\|^{2}+\left\|K_{n}^{+} f_{n k}\right\|^{2} \leq 2\left\|f_{n k}\right\|^{2} \leq 8 .
\end{gathered}
$$

Denote by $\mathcal{L}_{n}^{-}$the orthogonal complement of $\mathcal{L}_{n}^{+}$in the Krein space $\mathcal{K}_{n}$. Then $\mathcal{L}_{n}^{-}$is a maximal negative subspace of $\mathcal{K}_{n}$. The operator $K_{n}^{-}=\left(I_{n}-S_{n}\right)^{1 / 2}$, acting from $\mathcal{H}_{n}^{-}$into $\mathcal{H}_{n}^{+}$, is the angular operator of $\mathcal{L}_{n}^{-}$. The subspace $\mathcal{L}_{n}^{-}$is spanned by the vectors $\boldsymbol{f}_{n k}^{-}=\left(\begin{array}{c}K_{n}^{-} f_{n k} \\ f_{n k}\end{array}\right), k=1, \ldots, n$. This follows from the linear independence of $f_{n 1}, \ldots, f_{n n}$ and the relation

$$
\begin{aligned}
{\left[\boldsymbol{f}_{n j}^{+}, \boldsymbol{f}_{n k}^{-}\right] } & =\left(f_{n j}, K_{n}^{-} f_{n k}\right)-\left(K_{n}^{+} f_{n j}, f_{n k}\right) \\
& =\left(f_{n j},\left(I-S_{n}\right)^{1 / 2} f_{n k}\right)-\left(\left(I-S_{n}\right)^{1 / 2} f_{n j}, f_{n k}\right)=0 .
\end{aligned}
$$

The decomposition $\mathcal{K}_{n}=\mathcal{L}_{n}^{+}[\dot{+}] \mathcal{L}_{n}^{-}$is a fundamental decomposition of $\left(\mathcal{K}_{n},[\cdot, \cdot]\right)$. Solving a corresponding system of vector equations we find that the orthogonal (fundamental) projections $Q_{n}^{ \pm}$of the Krein space $\mathcal{K}_{n}$ onto $\mathcal{L}_{n}^{ \pm}$are given by

$$
Q_{n}^{+}=\left(\begin{array}{c}
I_{n} \\
K_{n}^{+}
\end{array}\right) S_{n}^{-1}\left(\begin{array}{ll}
I_{n} & -K_{n}^{-}
\end{array}\right), \quad Q_{n}^{-}=\left(\begin{array}{c}
K_{n}^{-} \\
I_{n}
\end{array}\right) S_{n}^{-1}\left(\begin{array}{ll}
-K_{n}^{+} & I_{n}
\end{array}\right) .
$$

From Lemma 1 it follows that $\left\|S_{n}^{-1}\right\|=n$. This and the above matrix representations of $Q_{n}^{ \pm}$imply that

$$
n \leq\left\|Q_{n}^{ \pm}\right\| \leq 2 n .
$$


Consequently, for any $\boldsymbol{f} \in \mathcal{K}_{n}$ we have

$$
\left\|Q_{n}^{ \pm} \boldsymbol{f}\right\| \leq 2 n\|\boldsymbol{f}\| .
$$

It follows from (5) that the vectors $\boldsymbol{f}_{n 1}^{+}, \ldots, \boldsymbol{f}_{n n}^{+}$form an orthonormal basis in the Hilbert space $\left(\mathcal{L}_{n}^{+},[\cdot, \cdot]\right)$. Denote by

$$
P_{n k}^{+}=\frac{\left[\cdot, \boldsymbol{f}_{n k}^{+}\right]}{\left[\boldsymbol{f}_{n k}^{+}, \boldsymbol{f}_{n k}^{+}\right]} \boldsymbol{f}_{n k}^{+}, \quad k=1, \ldots, n,
$$

the orthogonal projection in the Krein space $\mathcal{K}_{n}$ onto the subspace spanned by the vector $\boldsymbol{f}_{n k}^{+}, k=1, \ldots, n$. Then, by (5) and (6),

$$
1 \leq\left\|P_{n k}^{+}\right\|=\frac{\left\|\boldsymbol{f}_{n k}^{+}\right\|^{2}}{\left[\boldsymbol{f}_{n k}^{+}, \boldsymbol{f}_{n k}^{+}\right]} \leq 8, \quad k=1, \ldots, n
$$

Further, the operator

$$
J_{n 1}:=Q_{n}^{+}-Q_{n}^{-}
$$

is a fundamental symmetry in $\left(\mathcal{K}_{n},[\cdot, \cdot]\right)$. In particular, the inner product

$$
(\boldsymbol{x}, \boldsymbol{y})_{1}:=\left[J_{n 1} \boldsymbol{x}, \boldsymbol{y}\right], \boldsymbol{x}, \boldsymbol{y} \in \mathcal{K}_{n},
$$

is positive definite. Therefore, the operator $J_{n} J_{n 1}$ is positive and invertible in the Hilbert space $\left(\mathcal{K}_{n},(\cdot, \cdot)\right)$. Note also that $J_{n 1}=J_{n 1}^{-1}$. It follows from (8) that $\left\|J_{n 1}\right\|=\left\|J_{n 1}^{-1}\right\| \leq\left\|Q_{n}^{+}\right\|+\left\|Q_{n}^{-}\right\| \leq 4 n$. Consequently,

$$
\left\|J_{n} J_{n 1}\right\|=\left\|\left(J_{n} J_{n 1}\right)^{-1}\right\| \leq 4 n .
$$

The vectors $\boldsymbol{f}_{n j}^{+}, \boldsymbol{f}_{n k}^{-}, j, k=1, \ldots, n$, are orthonormal in $\left(\mathcal{K}_{n},(\cdot, \cdot)_{1}\right)$. This follows from (5), (7) and the relation

$$
\left(\boldsymbol{f}_{n j}^{+}, \boldsymbol{f}_{n k}^{-}\right)_{1}=\left[\left(Q_{n}^{+}-Q_{n}^{-}\right) \boldsymbol{f}_{n j}^{+}, \boldsymbol{f}_{n k}^{-}\right]=\left[Q_{n}^{+} \boldsymbol{f}_{n j}^{+}, \boldsymbol{f}_{n k}^{-}\right]=\left[\boldsymbol{f}_{n j}^{+}, \boldsymbol{f}_{n k}^{-}\right]=0 .
$$

Now we can apply Lemma 2 to the vectors $\boldsymbol{f}_{n j}^{+}, \boldsymbol{f}_{n k}^{-}, j, k=1, \ldots, n$, and the positive operator $J_{n} J_{n 1}$ : For given $\lambda_{1}^{ \pm}, \ldots, \lambda_{n}^{ \pm} \in \mathbb{C}$ define an operator $A_{n}$ by

$$
A_{n} \boldsymbol{f}_{n j}^{ \pm}=\lambda_{n j}^{ \pm} \boldsymbol{f}_{n j}^{ \pm}, j=1, \ldots, n,
$$

and then extend it by linearity to $\mathcal{K}_{n}$. It follows from Lemma 2 and (10) that

$$
\left\|A_{n}\right\| \leq 4 n \max \left\{\left|\lambda_{j}^{ \pm}\right|, j=1, \ldots, n\right\} \leq 4 C .
$$

Let $\mathcal{K}$ be the Krein space which is the direct orthogonal sum of the Krein spaces $\mathcal{K}_{n}, n \in \mathbb{N}$,

$$
\mathcal{K}:=\bigoplus_{n=1}^{\infty} \mathcal{K}_{n}
$$

The vectors $\boldsymbol{f}_{n j}^{ \pm}, j=1, \ldots, n, n \in \mathbb{N}$, constructed above are considered as elements of $\mathcal{K}$ and the Krein spaces $\mathcal{K}_{n}, n \in \mathbb{N}$, are considered as mutually orthogonal subspaces of $\mathcal{K}$. The vectors $\boldsymbol{f}_{n j}^{ \pm}, j=1, \ldots, n$, form a basis for $\mathcal{K}_{n}$. Let $\lambda_{n j}^{ \pm}, j=$ $1, \ldots, n$, be distinct real numbers such that $\pm \lambda_{n j}^{ \pm}>0, j=1, \ldots, n$, and such that there exists a constant $C$ with

$$
n \max \left\{\left|\lambda_{n j}^{ \pm}\right|, j=1, \ldots, n\right\} \leq C
$$

for all $n \in \mathbb{N}$. 
Put

$$
A:=\bigoplus_{n=1}^{\infty} A_{n}
$$

Then $A$ is a positive operator in the $\operatorname{Krein} \operatorname{space}(\mathcal{K},[\cdot, \cdot])$, and from (11) and (12) we get $\|A\| \leq 4 C$. Since the linear span of the vectors $\boldsymbol{f}_{n j}^{ \pm}, j=1, \ldots, n, n \in \mathbb{N}$, is dense in $\mathcal{K}$, it follows from the spectral theorem (see [1, Theorem IV.1.5] or [5] Theorem 3.1]) that the nonzero spectrum of $A$ consists of the simple eigenvalues $\lambda_{n j}^{ \pm}, j=1, \ldots, n, n \in \mathbb{N}$. Consequently, the left-hand side of the inequality (8) implies that 0 is a singular critical point of $A$ and the right-hand side of the inequality (9) implies that the norms of the orthogonal projections in $(\mathcal{K},[\cdot, \cdot])$ onto the eigenspaces of $A$ are uniformly bounded by 8 . The theorem is proved.

Remark. We can arrange the numbers $\lambda_{n j}^{+}, j=1, \ldots, n, n \in \mathbb{N}$, in a lower triangular table. Also, we can put the sequence $\left\{\frac{1}{m}, m \in \mathbb{N}\right\}$ in a lower triangular table by ending each row with a triangular number $\frac{n(n+1)}{2}$ in the denominator. A comparison of these two tables leads to

$$
\lambda_{n j}^{ \pm}:= \pm\left(\frac{n(n-1)}{2}+j\right)^{-1}, j=1, \ldots, n, n \in \mathbb{N} .
$$

In this way we get

$$
\left\{\lambda_{n j}^{ \pm}, j=1, \ldots, n, n \in \mathbb{N}\right\}=\left\{ \pm \frac{1}{m}, m \in \mathbb{N}\right\} .
$$

The numbers $\lambda_{n j}^{ \pm}$in (13) satisfy (12) with $C=2$. The proof of the Theorem implies that the nonzero spectrum of the operator $A$, which was constructed by means of the numbers $\lambda_{n j}^{ \pm}$from (13), consists of the simple eigenvalues $\pm \frac{1}{m}, m \in \mathbb{N}$.

If we consider the inverse $B=A^{-1}$ of the operator $A$ from the previous theorem and with the specific choice of numbers $\lambda_{n j}^{ \pm}$as in the Remark, we get:

Corollary. There exist a Krein space $(\mathcal{K},[\cdot, \cdot])$ and an unbounded positive operator $B$ in $\mathcal{K}$ with the following properties:

(a) The nonzero spectrum of $B$ consists of isolated simple eigenvalues.

(b) The point $\infty$ is a singular critical point of $B$.

(c) For each positive number $\mu$ we have

$$
\|E([a, b))\| \leq 8\lfloor\mu\rfloor \quad \text { whenever } \quad b-a<\mu,
$$

where $E$ is the spectral function of $B$ and $\lfloor\mu\rfloor$ denotes the largest integer smaller than $\mu$.

Proof. Let $A$ be the operator defined in the proof of the Theorem with the specific choice of the numbers $\pm \lambda_{n j}$ as in the Remark. Then $B=A^{-1}$ is a positive operator with a nonempty resolvent set (see e.g. [5, Proposition 3.1]), and $\sigma(B)=\mathbb{Z} \backslash\{0\}$. Let $\mu>0$ be arbitrary and let $0<b-a<\mu$. Then the interval $[a, b)$ contains at most $\lfloor\mu\rfloor$ eigenvalues of $B$. Therefore, $\|E([a, b))\| \leq 8\lfloor\mu\rfloor$. 


\section{REFERENCES}

1. T. Ya. Azizov, I. S. Iokhvidov, Linear operators in spaces with an indefinite metric. John Wiley \& Sons, New York, 1989. MR 90j:47042

2. B. Ćurgus, B. Najman, Quasi-uniformly positive operators in Krein spaces. Operator Theory and Boundary Eigenvalue Problems (Vienna, 1993), pp. 90-99, Oper. Theory: Adv. Appl., 80, Birkhäuser, Basel, 1995. MR 96j:47028

3. P. Jonas, Über die Erhaltung der Stabilität J-positiver Operatoren bei J-positiven und $J$ negativen Störungen. Math. Nachr. 65 (1975), 211-218. MR 53:3786

4. H. Langer, On maximal dual pairs of invariant subspaces of J-selfadjoint operators. Matem. Zametki 7 (1970), 443-447 (Russian). MR 42:3604

5. H. Langer, Spectral functions of definitizable operators in Krein spaces. Functional Analysis, Proceedings, Dubrovnik 1981. Lecture Notes in Mathematics 948, Springer-Verlag, Berlin, 1982, 1-46. MR 84g:47034

Department of Mathematics, Western Washington University, Bellingham, WashingTON 98225

E-mail address: curgus@cc.wwu.edu

Institutul de Matematică Al Academiei RomÂne, C.P. 1-764, 70700 BuCureß̧Ti, România

E-mail address: gheondea@imar.ro

Institute for Analysis, Vienna Technical University, Wiedner Hauptstrasse 8-10, A-1040 Vienna, Austria

E-mail address: hlanger@email.tuwien.ac.at 